\title{
Transport coefficients from in medium quarkonium dynamics
}

\section{Miguel A. Escobedo ${ }^{a, *}$}

${ }^{a}$ Instituto Galego de Física de Altas Enerxías, Universidade de Santiago de Compostela, E-15782 Galicia, Spain

E-mail: miguelangel.escobedo@usc.es

\begin{abstract}
Three are the mechanisms that influence quarkonium suppression in a medium: screening, thermal decay, and recombination. In recent years, a framework that can treat them consistently at the same time has been put forward, the open quantum system approach. In this talk, we will discuss how the combination of open quantum system and Effective Field Theory techniques are useful to understand quarkonium evolution in a medium in the regime in which the temperature is smaller than the inverse of the typical radius. In this case, the interaction parameter depends only on two transport coefficients $\kappa$ and $\gamma$, where $\kappa$ is the heavy quark diffusion coefficient. Combining these results with recent lattice QCD evaluations of the mass shift and thermal decay width of quarkonium we are able to obtain a non-perturbative determination of compatible with state-ofthe-art results. This talk is based on the recent paper [1].
\end{abstract}

HardProbes 2020

1-6 June 2020

Austin, Texas

${ }^{*}$ Speaker 


\section{Introduction}

Hard probes allow us to obtain information about the medium created in heavy-ion collisions. Because its production needs typically high energy, they are created at the beginning of the collision. However, they are modified substantially by the medium, and they are relatively easy to detect. In this work, we are going to focus on the hard probes related to heavy quarks.

- Heavy quark diffusion.

- Quarkonium suppression.

We will show that the heavy quark diffusion coefficient $\kappa$, which has an essential role in the physics of heavy quark diffusion, is also relevant for the study of quarkonium suppression. We will also discuss another transport coefficient related to the physics of quarkonium.

It is believed that heavy quarks in a quark-gluon plasma diffuse following approximately a Brownian motion, such that $\left\langle x^{2}(t)\right\rangle=6 D_{s} t$ (see [2] and references therein). This diffusion in coordinate space can be related to an analogous diffusion in momentum space characterized by the coefficient $\kappa=\frac{2 T^{2}}{D_{s}}$. An expression of $\kappa$ in terms of Wilson lines was found in [3]. In the following, we will discuss, among other things, how $\kappa$ also plays a role in the physics of quarkonium. The outline is the following:

- We will exploit the non-relativistic nature of quarkonium using Non-relativistic Effective Field Theories.

- Studying the evolution of the density matrix of heavy quarkonium in the regime $\frac{1}{r} \gg T \gg E$, we will derive an evolution equation in which all the information needed from the medium is encoded in two transport coefficients, $\kappa$ and $\gamma$.

- Using lattice QCD data of the decay width and thermal mass shift of quarkonium, we can extract a non-perturbative determination of $\kappa$ and $\gamma$.

\section{Non-relativistic Effective Field Theories to study quarkonium in a medium.}

Heavy quarkonium is a system in which a wide separation of energy scales appears. The mass of heavy quarks $m$ is much bigger than $\Lambda_{Q C D}$. Therefore, the creation or annihilation of heavy quarks is a perturbative process. In nowadays heavy-ion collisions, it also happens that $m \gg T$. There are other energy scales related to quarkonium's physics, the inverse of the typical radius $\frac{1}{r}$, and the binding energy $E$. In a non-relativistic bound state, it is fulfilled that $m \gg \frac{1}{r} \gg E$.

The appearance of widely separated energy scales is not just an academic issue. It can spoil the convergence of perturbative series even if the coupling constant is small, and, considering lattice QCD computations, it requires a huge lattice to fit all the relevant scales. These problems can be solved with the use of Effective Field Theories (EFTs). In the case of heavy quarks, the first step is to integrate out the mass of the heavy quarks. This can always be done using perturbative computations at $T=0$ since $m \gg \Lambda_{Q C D}, T$. In the case of quarkonium, the resulting EFT is called Non-relativistic QCD (NRQCD) [4, 5]. However, in NRQCD there are still at least two widely separated energy scales $\left(\frac{1}{r}\right.$ and $E$ ), and this can be problematic for some type of studies. In the 
zero temperature case, this can be solved by integrating out the scale $\frac{1}{r}$. This leads to potential NRQCD (pNRQCD) [6,7]. This EFT is equivalent to a potential model at leading order, but higher orders involve corrections that can not be mimic with a potential. The matching from NRQCD to pNRQCD transforms the relevant degrees of freedom from a heavy quark-antiquark pair to a color singlet field or a color octet field. At finite temperature, the relation between $\frac{1}{r}$ and $T$ can modify the matching between NRQCD and pNRQCD. This matching has been studied in a wide range of temperature regimes $[8,9]$.

\section{Open quantum system approach to quarkonium suppression}

The next question is how to use pNRQCD to compute the nuclear modification factor observed experimentally. Quarkonium is usually measured thought its decay into leptons. In pNRQCD, this is proportional to $\operatorname{Tr}\left(S^{\dagger}(t, \mathbf{0}) S(t, \mathbf{0}) \rho\right)$, where $S$ is the singlet field. Then, we need to solve this operator's evolution in a medium provided that we know its value at the time of the beginning of the fireball $t_{0}$. In fact, we can understand $\rho_{s}=\operatorname{Tr}\left(S^{\dagger}(t, \mathbf{0}) S(t, \mathbf{0}) \rho\right)$ as the reduced density matrix of heavy quarks when they are in a singlet state.

The evolution of the reduced density matrix was studied in [10] at several different temperature regimes. In the regime $\frac{1}{r} \gg T \gg E$ this evolution takes a particularly simple form

$$
\partial_{t} \rho=-i[H(\gamma), \rho]+\sum_{k}\left(C_{k}(\kappa) \rho C_{k}^{\dagger}(\kappa)-\frac{1}{2}\left\{C_{k}^{\dagger}(\kappa) C_{k}(\kappa), \rho\right\}\right),
$$

where the information about the medium in the operators $H(\gamma)$ and $C_{k}(\kappa)$ is encoded in two transport coefficients. The first one is the heavy quark diffusion parameter

$$
\kappa=\frac{g^{2}}{6 N_{c}} \operatorname{Re} \int_{-\infty}^{+\infty} d s\left\langle\mathrm{~T} E^{a, i}(s, \mathbf{0}) E^{a, i}(0, \mathbf{0})\right.
$$

and the second one is a new transport coefficient

$$
\gamma=\frac{g^{2}}{6 N_{c}} \operatorname{Im} \int_{-\infty}^{+\infty} d s\left\langle\mathrm{~T} E^{a, i}(s, \mathbf{0}) E^{a, i}(0, \mathbf{0})\right.
$$

In [10], we used the value of $\kappa$ obtained in the lattice computation of [11] and assumed that $\gamma=0$. Then, making some assumption about the initial density matrix of quarkonium and using a Bjorken evolution of the medium, we were able to make phenomenological predictions for the value of $R_{A A}$.

\section{Transport coefficients from in medium quarkonium dynamics}

We can now reverse the logic and determine $\kappa$ and $\gamma$ respectively from the decay width and the thermal mass shift. This is a useful strategy since there is unquenched lattice data on both the decay width and the thermal mass shift. In the case of $\kappa$, we have a determination which is based on an independent set of assumptions which can be compared with what is found studying heavy quark diffusion [2]. In the case of $\gamma$, it is the first non-perturbative determination. The main assumption is that we are in the regime $\frac{1}{r} \gg T, m_{D} \gg E$ and that the bound states are Coulombic. 

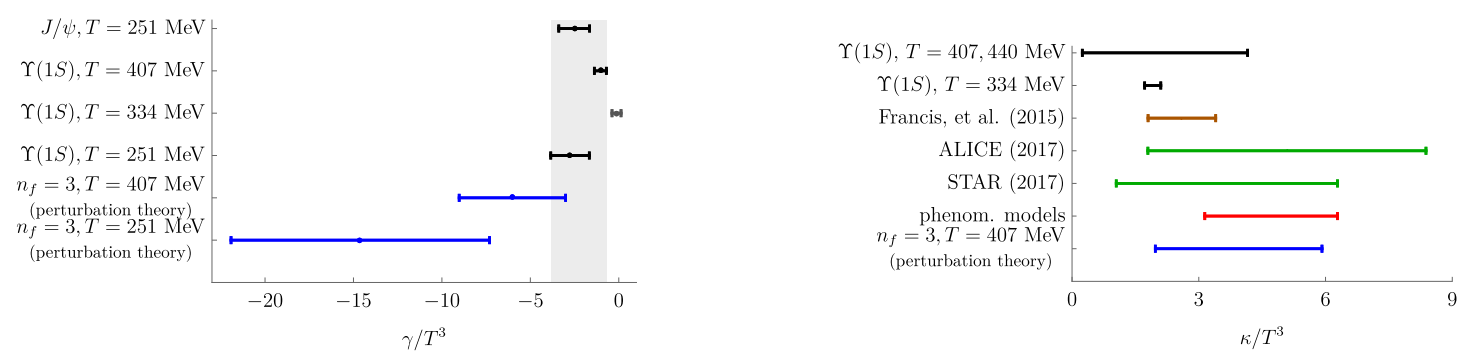

Figure 1: On the left, we show our determination of $\gamma$ using the mass shift data on different bound states at different temperatures. On the right, we show the same for $\kappa$ and compare with previous results in the literature. References to the literature can be found in [1]

The main formulas that we need are the following

$$
\delta M=\frac{1}{2} \gamma\left\langle r^{2}\right\rangle, \quad \Gamma=\kappa\left\langle r^{2}\right\rangle,
$$

where $\left\langle r^{2}\right\rangle$ can be computed analytically since we assume that the bound states are at leading order Coulombic. The lattice data that we are going to use in our analysis is the following

- We use the results of [12] for the thermal mass shift and as a lower bound for the decay width.

- We use the results of [13] as upper bound for the decay width.

- Data at $T=334 \mathrm{MeV}$, not used originally in [1], is taken from [14].

Fig. 1 summarises our results. Regarding $\gamma$, it is interesting to note that results from different quarkonium state at the same temperature $(T=251 \mathrm{MeV})$ are compatible with each other. There is also a hint that $\frac{\gamma}{T^{3}}$ is not a constant, as would be the case in the perturbative limit ignoring running coupling effects, or in a conformal theory. Finally, the values extracted from lattice data are much smaller that what one would naively expect from perturbative arguments. Regarding $\kappa$, our results are compatible with previous determinations.

\section{Conclusions}

We have reviewed the application of non-relativistic Effective Field Theories to the study of quarkonium suppression and its relation with the framework of open quantum systems. Using this formalism, we have used lattice data on the thermal mass shift and the decay width of quarkonium to determine two transport coefficients that are relevant to study quarkonium's evolution in a medium. One of these transport coefficients is $\kappa$, the heavy quark diffusion coefficient. Our determination of $\kappa$ is compatible with previous results in the literature. Regarding $\gamma$, we observe consistency between results obtained using data on different bound states and temperatures.

\section{Acknowledgements}

This work was supported by Ministerio de Ciencia e Innovacion of Spain under project FPA2017-83814-P and Maria de Maetzu Unit of Excellence MDM-2016- 0692, by Xunta de Galicia and FEDER. 


\section{References}

[1] N. Brambilla, M. A. Escobedo, A. Vairo and P. Vander Griend, Transport coefficients from in medium quarkonium dynamics, Phys. Rev. D 100 (2019) 054025 [1903. 08063].

[2] S. Cao et al., Toward the determination of heavy-quark transport coefficients in quark-gluon plasma, Phys. Rev. C 99 (2019) 054907 [1809. 07894].

[3] J. Casalderrey-Solana and D. Teaney, Heavy quark diffusion in strongly coupled $N=4$ Yang-Mills, Phys. Rev. D 74 (2006) 085012 [hep-ph/0605199].

[4] W. Caswell and G. Lepage, Effective Lagrangians for Bound State Problems in QED, QCD, and Other Field Theories, Phys. Lett. B 167 (1986) 437.

[5] G. T. Bodwin, E. Braaten and G. Lepage, Rigorous QCD analysis of inclusive annihilation and production of heavy quarkonium, Phys. Rev. D 51 (1995) 1125 [hep-ph/9407339].

[6] A. Pineda and J. Soto, Effective field theory for ultrasoft momenta in NRQCD and NRQED, Nucl. Phys. B Proc. Suppl. 64 (1998) 428 [hep-ph/9707481].

[7] N. Brambilla, A. Pineda, J. Soto and A. Vairo, Potential NRQCD: An Effective theory for heavy quarkonium, Nucl. Phys. B 566 (2000) 275 [hep-ph/9907240].

[8] N. Brambilla, J. Ghiglieri, A. Vairo and P. Petreczky, Static quark-antiquark pairs at finite temperature, Phys. Rev. D 78 (2008) 014017 [0804 . 0993].

[9] M. A. Escobedo and J. Soto, Non-relativistic bound states at finite temperature (I): The Hydrogen atom, Phys. Rev. A 78 (2008) 032520 [0804 . 0691].

[10] N. Brambilla, M. A. Escobedo, J. Soto and A. Vairo, Heavy quarkonium suppression in a fireball, Phys. Rev. D 97 (2018) 074009 [1711. 04515].

[11] A. Francis, O. Kaczmarek, M. Laine, T. Neuhaus and H. Ohno, Nonperturbative estimate of the heavy quark momentum diffusion coefficient, Phys. Rev. D 92 (2015) 116003 [1508.04543].

[12] S. Kim, P. Petreczky and A. Rothkopf, Quarkonium in-medium properties from realistic lattice NRQCD, JHEP 11 (2018) 088 [1808.08781].

[13] G. Aarts, C. Allton, S. Kim, M. Lombardo, M. Oktay, S. Ryan et al., What happens to the Upsilon and eta_b in the quark-gluon plasma? Bottomonium spectral functions from lattice QCD, JHEP 11 (2011) 103 [1109.4496].

[14] R. Larsen, S. Meinel, S. Mukherjee and P. Petreczky, Excited bottomonia in quark-gluon plasma from lattice QCD, Phys. Lett. B 800 (2020) 135119 [1910 . 07374]. 